\title{
Single Intrauterine Death in Twins: The Importance of Fetal Order
}

\author{
Penelope L. Ward, ${ }^{1}$ Karen L. Reidy, ${ }^{2}$ Ricardo Palma-Dias, ${ }^{2,3}$ Lex W. Doyle, ${ }^{3,4,5}$ and Mark P. Umstad ${ }^{3,6}$ \\ ${ }^{1}$ University of Melbourne, Faculty of Medicine, Dentistry and Health Sciences, Melbourne, Victoria, Australia \\ ${ }^{2}$ Pregnancy Research Centre, Department of Maternal Fetal Medicine and Department of Ultrasound, The Royal Women's \\ Hospital, Melbourne, Victoria, Australia \\ ${ }^{3}$ Department of Obstetrics and Gynaecology, The Royal Women's Hospital, University of Melbourne, Melbourne, Victoria, \\ Australia \\ ${ }^{4}$ Department of Paediatrics, The Royal Women's Hospital, University of Melbourne, Melbourne, Victoria, Australia \\ ${ }^{5}$ Clinical Sciences, Murdoch Childrens Research Institute, Melbourne, Victoria, Australia \\ ${ }^{6}$ Department of Maternal Fetal Medicine, The Royal Women's Hospital, Melbourne, Victoria, Australia
}

\begin{abstract}
Perinatal mortality is higher in twins. Effects of twin order have not previously been studied in the context of single fetal demise. Our objective was to determine whether death of the fetus more proximal to the cervix will result in worse perinatal outcomes. Our population included multiple pregnancies with two viable fetuses confirmed prior to 20 weeks' gestation with the subsequent death of at least one twin. All the pregnancies were managed at The Royal Women's Hospital, Melbourne between 2006 and 2014. We excluded pregnancies of higher order multiples, the death of both twins simultaneously, and cases with incomplete outcome data. Maternal and neonatal data were reviewed. Of 46 pregnancies included, in 24 (52\%), the dead twin was presenting. Gestational age at delivery was significantly earlier in these cases (mean difference: -5.0 weeks, $95 \% \mathrm{Cl}[-7.4,-2.6], \mathrm{p}<.001)$, and emergency cesarean rates were higher $67 \%$ versus $32 \%$ (OR 4.29, 95\% Cl $[1.25,14.7], p=.02$ ). There were no differences in the frequency of chorioamnionitis, preterm prelabor rupture of membranes, or placental abruption. Survival rates for co-twins were similar in both groups (presenting 83\%; not presenting 91\%; OR 0.41, 95\% $\mathrm{Cl}[0.07,2.50], p=.29$ ). The increase in neonatal morbidities was related to prematurity rather than to order. Findings were more common in dichorionic twins. Analysis was limited by a small sample size. If the dead twin is presenting, delivery is likely to occur earlier, with associated morbidity for the survivors. This is especially relevant for dichorionic twin pregnancies.
\end{abstract}

Keywords: death, fetal death, twins, multiple pregnancy, neonatal outcome, prognosis, twin order

Greater use of assisted reproductive techniques and delaying of pregnancy to more advanced maternal age have led to an increase in the number of multiple pregnancies (Umstad et al., 2013). Antenatal death of a single twin affects $15 \%$ of monochorionic (MC) and 3\% of dichorionic (DC) twin pregnancies after the first trimester (Hillman et al., 2011). Antenatal death is problematic for the surviving co-twin; for example, it can precede death of both twins (Hillman et al., 2010). Current research is focused on the neurological consequences for the viable twin and the long-term outcomes for these individuals (Jatzko et al., 2015; van Klink et al., 2015). Characterization of predictive factors that may influence morbidity and mortality can result in better counseling of parents on the prognosis for their remaining child.

Many studies describe the increase in morbidity for the second twin in uncomplicated twin pregnancies (Armson et al., 2006; Choi et al., 2015; Smith et al., 2007). Second twins experience greater rates of admission to neonatal intensive and special care units, respiratory distress, and mortality (Choi et al., 2015). The effect of twin order has not previously been studied in the context of single intrauterine fetal death (IUFD).

Our aim was to assess the effect of twin order in the context of single IUFD on perinatal outcomes, including the timing of delivery. We hypothesized that the death of

RECEIVED 4 July 2018; ACCEPTED 11 September 2018. First published online 22 October 2018.

ADDRESS FOR CORRESPONDENCE: Dr Penelope Ward, MD, University of Melbourne, Parkville, Victoria 3010, Australia. E-mail: penelopeleilaward@gmail.com 
the twin more proximal to the cervix would result in worse perinatal outcomes for the pregnancy and the surviving co-twin. The rationale behind this hypothesis is that the exposure of disintegrating tissue to external environments is potentially a risk factor for infection, as well as premature rupture of membranes and preterm labor.

\section{Materials and Methods}

This was a cohort study from a large tertiary referral center, The Royal Women's Hospital, Melbourne, Australia, of twin pregnancies with the loss of a single fetus later than 20 weeks' gestation over a 9-year period from 2006 to 2014. Cases of single fetal death were principally identified through the hospital's ultrasound Picture Archiving and Communication System GE Viewpoint. Additional cases were identified using the hospital inpatient classification system. Included cases were viable twin pregnancies at 20 weeks where there was a subsequent death of one fetus. We excluded multiple pregnancies of higher order, cases where the death of both twins was diagnosed simultaneously, and those where outcome data were unavailable or incomplete. Cases where fetal death was first diagnosed at delivery were also excluded.

The earliest ultrasound was used to determine the agreed date of delivery. Chorionicity was determined by the earliest available ultrasound prior to 20 weeks' gestation, and confirmed through placental histopathology after birth. Data were collected from maternal antenatal and inpatient records, as well as from neonatal records.

The study met the criteria established for quality assurance activities outlined in the National Health and Medical Research Council's guideline 'Ethical Considerations in Quality Assurance and Evaluation Activities' (National Health and Medical Research Council, 2014) and was approved as an audit by the Human Research Ethics Committee of The Royal Women's Hospital.

\section{Maternal Data}

Maternal parameters included age at pregnancy outcome, mode of conception, and comorbidities. Maternal complications including antenatal admission, selective fetocide date and indication, antenatal corticosteroid administration, and tocolysis were noted. Chorioamnionitis and placental abruption were defined by histopathological evidence. Cervical cerclage, gestational diabetes mellitus, twin-to-twin transfusion syndrome, and preterm prelabor rupture of membranes (PPROM) were recorded. Fetal growth restriction was diagnosed on antenatal fetal weight estimation and recorded for each twin separately, defined as an estimated fetal weight less than the 10th percentile using Hadlock 2 estimated fetal weight algorithm and reference charts. Intrapartum variables included the mode of delivery, presentation of each twin, duration and type of labor, and estimated blood loss. Gestation at IUFD and delivery was calculated.

\section{Neonatal Data}

Birth weight, sex, and neonatal outcomes were recorded for all twins. 1- and 5-minute Apgar scores and cord $\mathrm{pH}$ were recorded, where available. Admission to and durations of stay in the neonatal intensive care unit and the special care nursery were documented. Data were collected on the durations of assisted ventilation via an endotracheal tube and of nasal continuous positive airway pressure. Intraventricular hemorrhage, periventricular leukomalacia, bronchopulmonary dysplasia, necrotizing enterocolitis, and birth trauma were confirmed from available records. The presence of congenital and other anomalies was recorded for both the surviving and the index IUFD twin.

Twin order was determined through the review of ultrasound images and reports. The twin closer to the cervix at the time of IUFD diagnosis was considered to be presenting. We categorized pregnancies into two groups: IUFD Twin 1 where the dead twin was presenting, and IUFD Twin 2 where the surviving twin was presenting. The cause of death was determined by post-mortem examination, molecular karyotype, or imaging findings, when possible.

\section{Statistical Analysis}

The differences between groups for continuous variables were assessed using $t$ tests, and mean differences with 95\% confidence intervals (CI) were calculated; equal variances were not assumed if Levene's test suggested the nonhomogeneity of variance. Dichotomous variables were analyzed using either chi square or Fisher's exact test if sample sizes were small, and odds ratios (OR) with 95\% CI were calculated. To adjust for discrepancies in gestational age at delivery, continuous variables were analyzed by linear regression and dichotomous variables by logistic regression. IBM SPSS Statistics for Macintosh, Version 22.0 was used for data analysis.

\section{Results}

During the study period, 1,500 twin pregnancies were managed at the hospital, 58 of which met the initial inclusion criteria. Of these, 12 cases were excluded: 1 case was originally a triplet pregnancy, 3 were lacking outcome data, and 8 deaths were first diagnosed at delivery. A total of 46 cases were included in the study. In 24 (52\%) of these cases, the dead twin was presenting at the time of diagnosis.

\section{Maternal Demographic and Outcome}

Chorionicity, maternal age at pregnancy outcome, need for assisted reproductive techniques, and various obstetric complications were similar between the two groups 


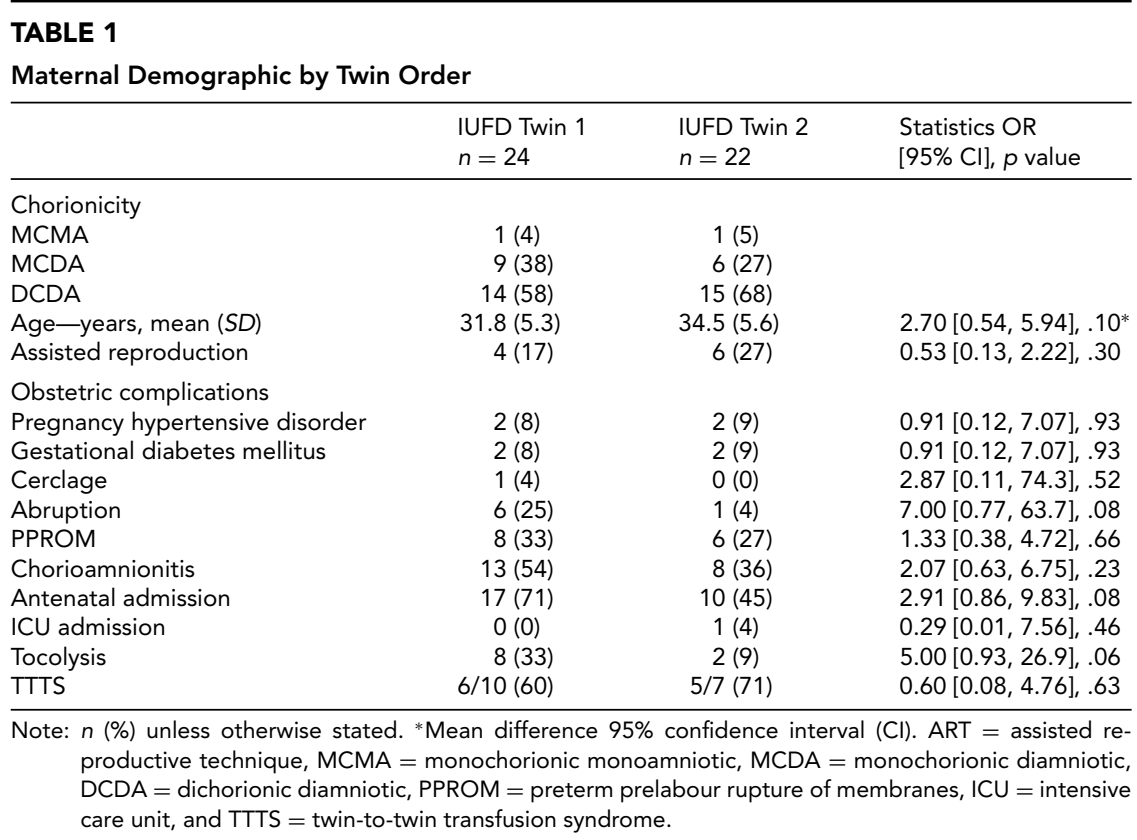

\section{TABLE 2}

\begin{tabular}{lc} 
Causes of Fetal Death & \\
\hline Cause of death & $n(\%)$ \\
\hline Structural or chromosomal anomaly & $15(32.6)$ \\
Twin-to-twin transfusion syndrome & $10 / 17(58.8)^{*}$ \\
Fetal growth restriction & $7(15.2)$ \\
Cord complication & $6(13.0)$ \\
Placental abruption & $4(8.7)$ \\
Chorioamnionitis & $2(4.3)$ \\
Cytomegalovirus infection & $1(2.2)$ \\
Unknown & $1(2.2)$ \\
\hline Note: *Percentage calculated from total monochorionic pregnancies.
\end{tabular}

(Table 1). In two cases, chorionicity was unable to be confirmed at placental histopathology; however, both of these were considered to be monochorionic diamniotic on ultrasound. There was no significant difference in the rates of PPROM, placental abruption, chorioamnionitis, or antenatal admission associated with twin order.

\section{Fetal Death}

Of the 46 cases, 12 had a selective reduction procedure of one twin because of chromosomal or structural anomalies, $42 \%(5 / 12)$ of which were presenting. In one case, the anomalies were associated with periconceptional cytomegalovirus infection. Structural or chromosomal anomalies were the most frequent cause of death overall (33\%) and accounted for 45\% (13/29) of index deaths in dichorionic diamniotic twins. Of all monochorionic diamniotic pregnancies, $59 \%$ (10/17) of deaths were caused by twin-to-twin transfusion syndrome. Other causes of death included cord complications, placental abruption, fetal growth restriction, and chorioamnionitis (Table 2). In one case, the cause of death was unknown after external post-mortem examination and failed molecular karyotype. The surviving co-twin in this case was found to have brain changes on magnetic resonance imaging consistent with congenital infection; however, maternal serology was negative.

\section{Obstetric and Intrapartum Outcomes}

The mean gestation at which the index fetal death occurred was 27.8 weeks and there was no significant difference between groups (Table 3). When the dead twin was presenting (IUFD Twin 1), delivery occurred at an average of 5 weeks earlier and the interval between diagnosis of index fetal death and delivery was on average 2.9 weeks shorter (Table 3).

Vaginal delivery and elective cesarean delivery rates were similar in the two study groups, whereas delivery by emergency cesarean section was more common when the dead twin was presenting (Table 3 ). In women who had an emergency cesarean, a higher proportion of those in the IUFD Twin 1 group experienced labor (Table 3). The rates of significant blood loss (greater than $500 \mathrm{~mL}$ ) were similar.

\section{Neonatal/Fetal Outcomes}

Of the 46 co-twin survivors of single IUFD, 40 (87\%) survived, $83 \%(20 / 24)$ in the IUFD Twin 1 group and $91 \%$ (20/22) in the IUFD Twin 2 group. Survival rates were not related to twin order (OR 0.41, 95\% CI [0.07, 2.50], $p=.29$ ). Five out of the six pregnancies where the co-twin died were monochorionic. Of the six deaths, one occurred in utero as a result of acute myeloid leukemia associated with trisomy 


\section{TABLE 3}

Obstetric and Intrapartum Outcomes by Twin Order at IUFD

\begin{tabular}{lccc}
\hline & $\begin{array}{l}\text { IIUFD Twin } 1 \\
n=24\end{array}$ & $\begin{array}{c}\text { IUFD Twin } 2 \\
n=22\end{array}$ & Statistics \\
\hline Obstetric outcomes & & Mean diff [95\% Cl], p value \\
Gest. age at IUFD (wks) & $26.8(3.0)^{*}$ & $28.9(5.5)^{*}$ & $-2.2[-4.8,-0.5], .11^{* *}$ \\
Gest. age at delivery (wks) & $29.0(2.9)^{*}$ & $34.1(4.8)^{*}$ & $-5.0[-7.4,-2.6],<.001^{* *}$ \\
IUFD to delivery interval (wks) & $2.3(2.6)^{*}$ & $5.2(4.9)^{*}$ & $-2.9[-5.2,-0.6], .016$ \\
Intrapartum outcomes & & & OR [95\% Cl], p value \\
Vaginal delivery & $6(25)$ & $7(36)$ & $0.58[0.16,2.07], .40$ \\
Elective cesarean & $2(8)$ & $7(32)$ & $0.19[0.03,1.07], .06$ \\
Emergency cesarean & $16(67)$ & $7(32)$ & $4.29[1.25,14.7], .02$ \\
$\quad$ In labor & $12 / 16(75)$ & $2 / 7(29)$ & $7.50[1.02,55.0], .05$ \\
Significant blood loss & $8(33)$ & $9(41)$ & $0.72[0.22,2.40], .59$ \\
\hline Note: $n$ (\%) unless otherwise stated. & & & \\
$*$ *mean (SD). & &
\end{tabular}

21, which affected both twins. The chromosomal anomaly and the hematological diagnosis were confirmed at fetal blood sampling during intrauterine transfusion of the surviving co-twin, who then died 4 days later. The other five deaths occurred after birth.

In the first case, ventriculomegaly was diagnosed antenatally following co-twin death from cord entanglement in a monoamniotic twin pregnancy. Delivery was at $31+$ 6 weeks. After hypoxic ischemic encephalopathy, and confirmation of extensive periventricular leukomalacia on cranial ultrasound, the infant received palliative care.

The second case was a survivor in a case of twin-anemia polycythemia sequence. The neonate was given a poor prognosis, and labor was induced at $26+4$ weeks with a plan for palliation.

The third case of a neonatal death was in a dichorionic diamniotic survivor born at $24+2$ weeks whose management was changed to palliation on day 22 of life due to severe cortical cystic brain infarction.

In the fourth case, where birth occurred at 23 weeks, there was antenatal detection of ventriculomegaly and intraventricular hemorrhage, and the infant received palliative care after birth.

The fifth neonatal death was a consequence of extreme prematurity, with delivery at 23 weeks and 2 days. Examination of the placenta indicated that preterm labor was likely related to a moderately large retroplacental hemorrhage.

There were significantly more anomalies detected in surviving twins where the dead twin had been presenting (Table 4). In total, $70 \%$ of the liveborn co-twins in the IUFD Twin 1 group were found to have an anomaly that was either congenital or acquired, compared with only $9 \%$ in the IUFD Twin 2 group (OR 22.9, 95\% CI [4.2, 125.6], $p=.01$ ). In 11 cases, there were anomalies present in both the surviving twin, and their co-twin who had died. Five of these cases showed concordant or related anomalies, four in the IUFD Twin 1 group and one in the IUFD Twin 2 group. The remaining six cases of discordant fetal anomalies were all in IUFD Twin 1.
Univariable analysis demonstrated a significant difference between the birth weights of co-twins born alive when comparing those that were presenting with those that were not at the time of death of their co-twin (Table 4). Regression analysis demonstrated that earlier delivery was the main contributor to this discrepancy, and twin order alone was not associated with birth weight. Likewise, 1-minute Apgar scores were significantly lower due to younger gestational age at delivery rather than twin order. Other neonatal parameters that were significant with univariable analysis were also found to be attributable to earlier delivery within the IUFD Twin 1 group.

\section{Effect of Chorionicity}

Mean gestational age at delivery was 32.8 weeks in DC pregnancies, which was significantly higher than the 29.1 weeks seen in MC pregnancies (mean difference: 3.7 weeks, $95 \%$ CI $[1.0,6.3], p=.01)$. Subgroup analysis of the DC and MC pregnancies revealed that the differences observed in gestational age at delivery and neonatal outcomes due to twin order were only present in DC twins. For DC twins, mean gestation at delivery was 6.8 weeks earlier in the IUFD Twin 1 group (95\% CI [4.5, 9.1], $p<.01)$. The gestational age at delivery for MC twins was not related to twin order (mean difference: $2.6,95 \%$ CI $[1.7,6.9], p=.2$ ). In the DC group where the dead twin was presenting, chorioamnionitis was significantly more common (OR 6.88, 95\% CI $[1.35,35.1]$, $p=.02$ ), as were antenatal admissions (OR 19.5, 95\% CI $[2.0,190], p=.01)$ and emergency cesarean delivery (OR $5.00,95 \%$ CI $[1.03,25.3], p=.046)$. For the surviving cotwins of DC pregnancies, the IUFD Twin 1 group had comparatively more neonatal nursery admissions (OR 24.4, 95\% CI $[2.13,84.3], p=.01$ ), respiratory distress (OR 14.7, 95\% CI $[2.4,88.5], p<.01)$, and treatment with nasal continuous positive airway pressure (OR 6.9, 95\% CI [1.4, 35.1], $p=.02)$. There were no significant differences between the outcomes for mothers or surviving co-twins when comparing twin order within the MC group. 


\section{TABLE 4}

Liveborn Twin Outcomes by Twin Order

\begin{tabular}{|c|c|c|c|c|}
\hline Neonatal variables & $\begin{array}{l}\text { IUFD Twin } 1 \\
n=23\end{array}$ & $\begin{array}{l}\text { IUFD Twin } 2 \\
n=22\end{array}$ & $\begin{array}{l}\text { Univariable } \\
\text { ( } p \text { value) }\end{array}$ & $\begin{array}{l}\text { Multivariable } \\
\text { ( } p \text { value) }\end{array}$ \\
\hline Anomalies in survivor, $n(\%)$ & $16(70)$ & $2(9)$ & $<.01$ & $<.01$ \\
\hline Birthweight, mean $(S D)$ & $1,224(497)$ & 2,206 (964) & $<.01$ & .39 \\
\hline \multicolumn{5}{|l|}{ Apgar scores } \\
\hline 1 minute, median (IQR) & $6.0(3.8-8.0)$ & $8(6.8-9.0)$ & .02 & .40 \\
\hline 5 minute, median (IQR) & $8.0(7.0-9.0)$ & $9(8.0-9.0)$ & .05 & .65 \\
\hline \multicolumn{5}{|l|}{ Cord $\mathrm{pH}(n=23)$} \\
\hline Venous, mean $(S D)$ & $7.30(0.07)$ & $7.32(0.16)$ & .23 & .33 \\
\hline Arterial, mean (SD) & $7.26(0.10)$ & $7.25(0.18)$ & .13 & .30 \\
\hline NISC admission, $n$ (\%) & $21(91)$ & $11(50)$ & $<.01$ & .05 \\
\hline Total days, median (IOR) & $32(17-51)$ & $17(4-63)$ & .39 & .06 \\
\hline NICU admission, $n(\%)$ & $17(74)$ & $7(32)$ & $<.01$ & .08 \\
\hline NICU hours, median (IQR) & $226(30-494)$ & $31(0-274)$ & .40 & .12 \\
\hline $\mathrm{SCN}$ admission, $n(\%)$ & $20(87)$ & $11(50)$ & .01 & .06 \\
\hline SCN days, median (IOR) & $20(8-29)$ & $11(3-21)$ & .60 & .55 \\
\hline \multicolumn{5}{|l|}{ Total care days $(n=33)$} \\
\hline Median (IQR) & $49(32-81)$ & $17(6-67)$ & .08 & .39 \\
\hline Respiratory distress, $n(\%)$ & $17(74)$ & $7(32)$ & $<.01$ & .18 \\
\hline Surfactant, $n(\%)$ & $7(30)$ & 1 (4) & .05 & .19 \\
\hline Intubation, $n(\%)$ & $7(30)$ & $2(9)$ & .09 & .53 \\
\hline CPAP, $n(\%)$ & $16(70)$ & $8(36)$ & .03 & .20 \\
\hline CPAP hours, median (IQR) & $120(38-277)$ & $21(9-277)$ & .54 & .10 \\
\hline
\end{tabular}

Note: $†$ Adjusted for gestational age at delivery. GA = gestational age, NISC = neonatal intensive and special care, $\mathrm{NICU}=$ neonatal intensive care unit, $\mathrm{SCN}=$ special care nursery, and $\mathrm{CPAP}=$ continuous positive airway pressure.

\section{Discussion}

The major finding of our study of twin pregnancies complicated by a single fetal death after 20 weeks was that delivery occurred significantly earlier when the dead twin was presenting. Earlier delivery resulted in more neonatal morbidity for the surviving co-twin, with higher rates of admission to intensive care for respiratory support to treat respiratory distress. After adjusting for differences in gestational age, birth order itself did not affect neonatal morbidity. The other major finding was that the rate of anomalies in the surviving co-twin was much higher if it was the presenting twin who had died. In addition, maternal morbidity was largely unrelated to the order of fetal death, although emergency cesarean delivery was more common with IUFD Twin 1.

We had hypothesized worse outcomes for surviving twins related to chorioamnionitis and PPROM as a disintegrating fetus is more likely to be a source for infection if it is closer to the cervix. A loss of tissue integrity could also cause the rupture of membranes, abruption, and labor. The higher frequencies of abruption and chorioamnionitis were not statistically significant between groups and may represent a beta error because our study was small. Nonetheless, we are yet to identify a definitive cause for the observed relative prematurity.

It is recognized that anomalies are present at higher rates in twins (Sperling et al., 2007). Our study was no exception, showing structural and chromosomal abnormalities to be a major cause of fetal death, either because of lethal changes or selective termination. A total of $40 \%$ of surviving twins were also affected by anomalies. An unexpected finding was the high rate of anomalies affecting surviving co-twins when fetal death occurred in Twin 1, which is unexplained by current theories on high rates of anomalies in twins.

Many studies have demonstrated worse outcomes for the second twin (Choi et al., 2015; Smith et al., 2002, 2007). There are a number of theories as to why the second twin born has an inferior outcome when compared with the first. It has been speculated that asphyxia due to prolonged intertwin delivery time and malpresentation both contribute to worse outcomes in twins (Arnold et al., 1987), and despite the development of antenatal corticosteroids, postnatal surfactant, and modern intensive care, there remains a clear disadvantage for the second-born twin (Shinwell et al., 2004). Our results demonstrate that this is the case even when intrauterine death of a single twin occurs, because of the higher risk of prematurity when the dead twin is presenting.

Chorionicity is known to be the best predictor of outcomes for the survivor of an IUFD in twins, due to the consequences of shared placental circulation (Hillman et al., 2010). A recent study showed a difference in the rate of preterm delivery between MC and DC groups to be $68 \%$ and 54\%, respectively (Hillman et al., 2011). Our results were consistent with this finding, demonstrating a statistically significant difference in gestation at delivery between $\mathrm{MC}$ and DC twin in cases of single fetal death.

\section{Strengths and Limitations}

Fortunately, the single death of a twin is a relatively rare event. Our study was undertaken at a large tertiary 
referral center over a 9-year period in which 1,500 twin pregnancies were delivered. Even so, a limitation of our study is the low number of cases. Inclusion of pregnancies where death was the result of selective fetocide after 20 weeks' gestation increased the study numbers. However, the controlled nature of these events may mean that outcomes are more favorable for this group (Cleary-Goldman \& D'Alton, 2004). This as well as the skew towards anomalies in IUFD Twin 1 survivors mitigate the generalizability of our research on a larger scale. Research on single IUFD is moving towards separate analysis of the outcomes following selective reduction because of this difference in prognosis for the surviving twin (O'Donoghue et al., 2009). Regression analysis was similarly limited by low participant numbers. Adjustment for gestational age was done for all cases, which allowed us to show that the difference in outcomes for the survivor was largely related to gestational age rather than twin order. With only 29 DC pregnancies, it was not possible to do the same adjustment within this group alone, where gestational age at delivery was seen to be significantly less when the dead twin was presenting. Given the well-described influence of chorionicity on pregnancy outcomes in single IUFD, this inability to analyze groups separately is a significant limitation to our study.

\section{Interpretation}

Our study helps to advise pediatric clinicians and parents of the risks to the surviving child, and assists in identifying those cases with a higher risk of neonatal morbidity due to early gestational age at delivery. Medicalization of the neonatal period can be tumultuous for parents, adding to the emotional stress of losing a child. Parents also report impaired decision making when there is single fetal loss in a multiple pregnancy (Richards et al., 2015), something to which clinicians should be sensitive. Counseling on expected outcomes for the pregnancy, including the risk of early delivery, may help parents to prepare themselves during what is likely to be a difficult time.

\section{Conclusions}

In our study, we have demonstrated that in twin pregnancies with single IUFD, there may be worse outcomes for the pregnancy and survivor if the dead twin is presenting. Gestation at delivery is significantly earlier, and because of this, the complications of prematurity are more prevalent. Those of dichorionic placentation are particularly affected by twin order. More research should be undertaken to establish the mechanism behind early delivery, and the role of chorioamnionitis in these outcomes.

\section{Acknowledgments}

Study data were collected and managed using REDCap electronic data capture tools hosted at The University of
Melbourne (Harris et al., 2009). The first author was supported as a postgraduate student by the University of Melbourne.

\section{Disclosure of Interest}

In accordance with the University of Melbourne, The Royal Women's Hospital Pregnancy Research Centre, and Division of Maternity Services policies and procedures, as well as my ethical obligation as a researcher, I am reporting that the authors have no potential conflicts of interest to declare. Study design, collection and analysis of data, and report writing were supported by university facilities and staff, but driven by supervisors from The Royal Women's Hospital Pregnancy Research Centre and the Division of Maternity Services, who acted in a teaching capacity.

\section{Details of Ethical Approval}

The study met the criteria established for quality assurance activities outlined in the National Health and Medical Research Council's guideline 'Ethical Considerations in Quality Assurance and Evaluation Activities' and was approved as an audit by the Human Research Ethics Committee of The Royal Women's Hospital on August 25, 2015.

\section{References}

Armson, B. A., O’Connell, C., Persad, V., Joseph, K. S., Young, D. C., \& Baskett, T. F. (2006). Determinants of perinatal mortality and serious neonatal morbidity in the second twin. Obstetrics \& Gynecology, 108, 556-564.

Arnold, C., McLean, F. H., Kramer, M. S., \& Usher, R. H. (1987). Respiratory distress syndrome in second-born versus first-born twins. A matched case-control analysis. New England Journal of Medicine, 317, 1121-1125.

Choi, S.-J., Kim, S. H., Lee, H.-J., Kim, M., Sung, J.-H., Oh, S.-Y., ... Kim, J.-H. (2015). 555: Effect of birth order on neonatal outcome of twins according to gestational age at delivery: Early preterm vs late preterm vs term. American Journal of Obstetrics \& Gynecology, 212, S277.

Cleary-Goldman, J., \& D’Alton, M. (2004). Management of single fetal demise in a multiple gestation. Obstetrical \& Gynecological Survey, 59, 285-298.

Harris, P. A., Taylor, R., Thielke, R., Payne, J., Gonzalez, N., \& Conde, J. G. (2009). Research electronic data capture (REDCap) - A metadata-driven methodology and workflow process for providing translational research informatics support. Journal of Biomedical Informatics, 42, 377381.

Hillman, S. C., Morris, R. K., \& Kilby, M. D. (2010). Single twin demise: Consequence for survivors. Seminars in Fetal \& Neonatal Medicine, 15, 319-326.

Hillman, S. C., Morris, R. K., \& Kilby, M. D. (2011). Co-twin prognosis after single fetal death: A systematic review and meta-analysis. Obstetrics \& Gynecology, 118, 928-940.

Jatzko, B., Rittenschober-Bohm, J., Mailath-Pokorny, M., Worda, C., Prayer, D., Kasprian, G., \& Worda, K. (2015). 
Cerebral lesions at fetal magnetic resonance imaging and neurologic outcome after single fetal death in monochorionic twins. Twin Research and Human Genetics, 18, 606612.

National Health and Medical Research Council. (2014). Ethical considerations in quality assurance and evaluation activities. Retrieved from https://www.nhmrc.gov.au/ guidelines-publications/e111

O’Donoghue, K., Rutherford, M. A., Engineer, N., Wimalasundera, R. C., Cowan, F. M., \& Fisk, N. M. (2009). Transfusional fetal complications after single intrauterine death in monochorionic multiple pregnancy are reduced but not prevented by vascular occlusion. BJOG, 116, 804-812.

Richards, J., Graham, R., Embleton, N. D., Campbell, C., \& Rankin, J. (2015). Mothers' perspectives on the perinatal loss of a co-twin: A qualitative study. BMC Pregnancy Childbirth, 15, 143.

Shinwell, E. S., Blickstein, I., Lusky, A., \& Reichman, B. (2004). Effect of birth order on neonatal morbidity and mortality among very low birthweight twins: A population based study. Archives of Disease in Childhood: Fetal \& Neonatal Edition, 89, F145-148.
Smith, G. C., Fleming, K. M., \& White, I. R. (2007). Birth order of twins and risk of perinatal death related to delivery in England, Northern Ireland, and Wales, 1994-2003: Retrospective cohort study. BMJ, 334, 576.

Smith, G. C., Pell, J. P., \& Dobbie, R. (2002). Birth order, gestational age, and risk of delivery related perinatal death in twins: Retrospective cohort study. BMJ, 325, 1004.

Sperling, L., Kiil, C., Larsen, L. U., Brocks, V., Wojdemann, K. R., Qvist, I., ... Tabor, A. (2007). Detection of chromosomal abnormalities, congenital abnormalities and transfusion syndrome in twins. Ultrasound in Obstetrics and Gynecology, 29, 517-526.

Umstad, M. P., Hale, L., Wang, Y. A., \& Sullivan, E. A. (2013). Multiple deliveries: The reduced impact of in vitro fertilisation in Australia. Australian and New Zealand Journal of Obstetrics and Gynaecology, 53, 158-164.

van Klink, J. M., van Steenis, A., Steggerda, S. J., Genova, L., Sueters, M., Oepkes, D., \& Lopriore, E. (2015). Single fetal demise in monochorionic pregnancies: Incidence and patterns of cerebral injury. Ultrasound in Obstetrics and Gynecology, 45, 294-300. 